\title{
Pathogenesis of the acute coronary syndromes and therapeutic implications
}

\author{
L. Badimon '; J.J. Badimon 2; G. Vilahur '; E. Segalés '; V. Llorente ${ }^{1}$ \\ ${ }^{1}$ Cardiovascular Research Center, ICCC-CSIC, Hospital de la Santa Creu i Sant Pau, Barcelona (Spain) and ${ }^{2}$ Cardiovascular Institute, Mount Sinai Medical \\ Center, New York, (USA)
}

\section{Key Words}

Atherosclerosis, thrombosis, statins, fibrinolytics, heparin, anti-platelet agents, atherosclerotic plaque, unstable angina, myocardial infarction.

\begin{abstract}
Atherosclerosis is characterized by the thickening and obstruction of the arterial lumen and thrombosis associated with vulnerable disrupted plaques seems to be responsable for the accelerated process of clinical sindrome presentation.

Strategies to promote plaque stabilization and reduce thrombus burden have been one of the major targets of recent times. Modification of diet and lifestyle has important benefit in reducing coronary risk. Several pharmacological strategies for reducing cardiovascular morbidity and mortality have demonstrated effectiveness. Statins and angiotensin converting enzyme, fibrinolitics, inhibitors of the intrinsic coagulation cascade and anti-platelet agents have been developed to reduce the impact of atherosclerosis and inhibit thrombogenesis.
\end{abstract}

Copyright @ 2002 S. Karger AG. Basel

\section{Introduction}

Atherosclerotic disease is a focal pathological phenomena characterized by the thickening and obstruction of the arterial lumen. Epidemiological, clinical and experimental studies have identified several risk factors that are relevant in patients with atherosclerotic disease. Thrombosis on vulnerable high risk plaques is the major trigger of acute ischemic events in the coronary, cerebral and peripheral arterial circulation.

Endothelial dysfunction is one of the initial targets and

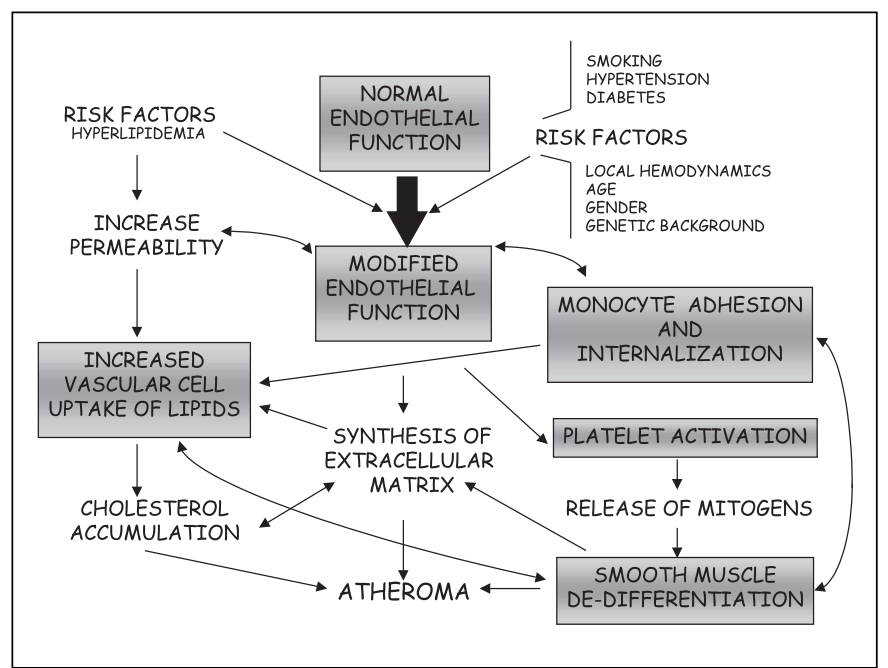

Fig. 1. Simplified diagram of atherosclerotic plaque development.

Prof. Lina Badimon

CIC / CSIC

Jordi Girona, 18-26 - 08034 Barcelona, Spain

Tel: 34934006146

Fax: 34932045904

e-mail : lbmucv@cid.csic.es 
triggers of the successive events responsible for the formation of atherosclerotic lesions [1-4] (Figure 1). The clustering of risk factors (elevated LDL-cholesterol, smoking, diabetes, high blood pressure and low HDL-cholesterol levels) and their local hemorheological, hemodynamic and biochemical effects synergically contribute to the induction of the initial primary lesion. A break in the endothelial permeability barrier will facilitate the entrance of circulating monocytes and plasma lipids into the arterial wall, as well as platelet deposition at the sites of endothelial denudation. Damaged endothelial cells, monocytes, and aggregated platelets through the release of mitogenic factors potentiate the migration and proliferation of vascular SMC, that together with increased receptor-mediated lipid accumulation and increased connective tissue synthesis, would shape the typical atheromatous plaque. Perpetuation of these processes may account for the slow progression of the disease. However, in some instances a much faster development is observed; thrombosis associated with vulnerable disrupted plaques seems to be responsible for the accelerated process of clinical syndrome presentation.

\section{Evolution of atherothrombosis}

\section{The initial lesion}

Atherosclerotic lesions tend to develop in areas which are under repeated mechanical force changes, such as oscillating shear forces. The endothelium at these sites is characterized by increased permeability leading to an influx of low-density lipoproteins (LDL) and other plasma proteins into the intima. Additionally, circulating monocytes adhere to activated endothelial cells (expressing adhesive cell-surface glycoproteins such as E-selectin, VCAM-1 or ICAM-1), migrate between endothelial cells, enter the intima and differentiate into macrophages which can endocytose native and oxidized LDL. Lipids can also accumulate indirectly after death of lipid-rich foam cells [5] (Table 1).
The lipid rich core originates primarily from lipoprotein trapping and binding to matrix proteins such as glycosaminoglycans, collagen and fibrinogen; this process results in focal collections of lipid laden foam cells seen microscopically as yellow fatty clots or streaks raised above the intimal surface. Recently, an important function in intracellular lipid accumulation has been recognized for LRP in vascular smooth muscle cells [6-7]. Indeed aggregated, versican-fused-LDL are avidly taken up by human smooth muscle cells [8] and the gene codifying for the receptor is even up-regulated in the presence of high levels of LDL [9].

The initial lipid and macrophage driven process is subsequently accompanied by smooth muscle cell activation, migration, and proliferation, followed by extracellular matrix deposition and further lipid accumulation. This gives rise to more mature and clinically significant atherosclerotic plaques. The endothelium is often physically and functionally impaired by further recruitment of monocytes and macrophages, compounded by the toxic effects of oxidized LDL, and degradation products such as oxygen free-radicals (Figure 2 and Figure 3, upper pannel).

\section{The high risk vulnerable plaques}

Atherosclerosis is a multifocal disease, the coronary arteries are generally diffusely affected with confluent plaques carpeting the vessel wall, but individual plaques vary greatly in composition. In patients with acute coronary syndromes, a significant atheromatous core is present in the majority of unstable plaques. Fibrous plaques are stable and often resistant to disruption, but may produce stable angina if coronary blood flow is reduced.

A vulnerable fibrolipid plaque consists of a lipid-rich core separated from the arterial lumen by a fibromuscular cap. This atheromatous core is mostly avascular, hypocellular (except at the periphery where macrophage foam cells are frequently present), devoided of supporting collagen, rich in free cholesterol esters and very soft [10]. The cap-region of the plaque is

Table 1. Endothelium and cardiovascular homeostasis factors.

\begin{tabular}{ll}
\hline ATHEROGENIC & ATHEROPROTECTIVE \\
\hline VASOCONSTRICTORS & VASODILATORS \\
$\begin{array}{l}\text { Angiotensin II, Endothelin, Thromboxane, } \\
\text { Serotonin, Thrombin, Nicotine }\end{array}$ & Nitric oxide, Prostacyclin, Histamine, \\
SMC PROMOTERS & Bradykinin, Serotonin, Substance P \\
PDGF, bFGF, Endothelin, Angiotensin II & SMC INHIBITORS \\
PROTHROMBOTIC & Nitric oxide, Prostacyclin, Bradykinin, TGF $\beta$ \\
Thromboxane, Thrombin, vWF & ANTITHROMBOTIC \\
PRO-INFLAMMATORY & Thrombomodulin, TFPI, $\uparrow$ tPA, $\downarrow$ PAI-1 \\
VCAMs, ICAMs, ELAMs, Selectins & ANTI-INFLAMMATORY \\
\hline BFGF $=$ basic fibroblast growth factor; ELAMs $=$ endothelial leukocyte adhesion molecules; ICAMs $=$ intracellular adhesion molecules; PAI-1 $=$ pla- \\
sminogen activator inhibitor $1 ;$ PDGF $=$ platelet-derived growth factor; SMC $=$ smooth muscle cell; TFPI $=$ tissue factor platelet inhibito; TGF- $\beta=$ \\
transforming growth factor- $-\beta$; t-PA $=$ tissue plasminogen activator; VCAMs $=$ vascular cell adhesion molecules; vWF $=$ von Willebrand Factor
\end{tabular}




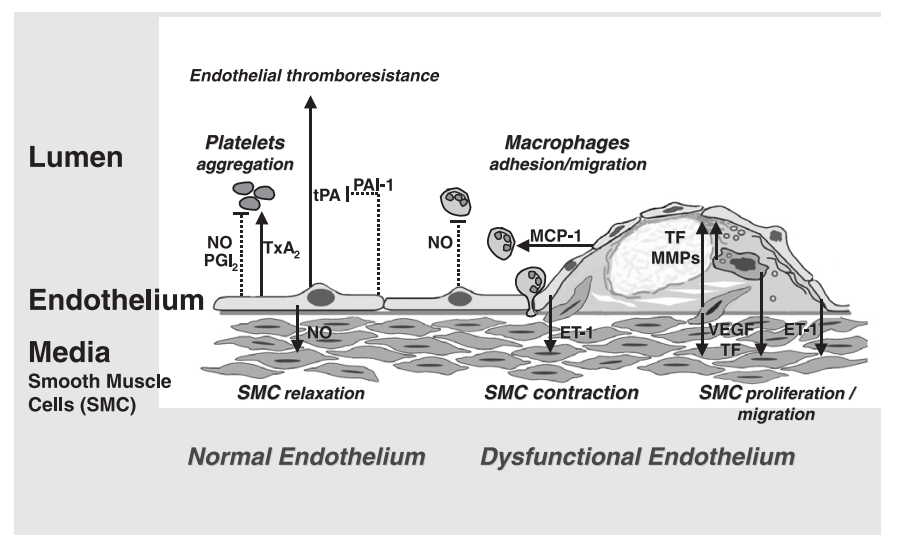

Fig. 2. Endothelium, antiatherogenic and antithrombotic properties. The endothelium plays a pivotal role in the vascular homeostasis by regulating vascular tone and blood thrombogenicity. Schematic of the multiple function of the endothelium in the regulation of platelet function, blood thrombogenicity, smooth muscle cells (SMC) proliferation and migration, and vasomotion. Normal functioning endothelium (left) produces several substances aimed to maintain normal shear condition by balancing the production of vasodilator $(\mathrm{NO})$ and vasoconstrictor (ET-1), avoids excessive platelet aggregation (TFPI/TF) and fibrinolysis (tPA/PAI-1). Dysfunction endothelium (right) favors macrophage adhesion and migration (MCP-1), plaque growth and induces vasoconstriction.

also subjected to peak hemodynamic stresses. These are the high-risk plaques, soft, unstable and vulnerable, that are prone to rupture. For eccentric plaques, unstability is often observed in the shoulder region at the junction of the plaque with the less diseased adjacent vessel wall (Figure 3).

The atheromatous gruel is the most thrombogenic component of a human plaque [11] and its high thrombogenicity is due to tissue factor presence in the plaque boundary layer [12]. The presence of tissue factor in plaques is clearly documented [13] although it is not yet known when and how its expression is induced. Therefore, nonstenotic but soft plaques should be considered ominous since myocardial infarction may often result from acute occlusion related to disrupted lipid-laden lesions. These observations suggest that limiting the management of coronary artery disease to severely stenotic atherosclerotic plaques may not entirely prevent ACS and sudden death.

\section{Acute coronary syndromes}

Strategies addressed to promote plaque stabilization and reduce thrombus burden have been one of the major targets of recents times.

Modification of diet and lifestyle has tremendous benefit in reducing coronary risk. Recently, a large Finnish study [14] demonstrated the role of lifestyle modification in reducing the progression of prediabetic patients into frank diabetes. This effect on reducing diabetic progression may translate into a reduction in the occurrence of plaque disruption.



Fig. 3. Histological analysis of human coronary arteries (Masson Trichromic Stain) from intimal thickening (upper) to high risk plaque (middle), to ruptured plaque with thrombus (lower panel).

Several pharmacological strategies with the ultimate goal of reducing cardiovascular morbidity and mortality have demostrated effectiveness. Among them, statins and angiotensin converting enzyme inhibitors have clearly demonstrated significant clinical benefit, which has been in part explained through plaque stabilization.

\section{Statins}

Statin therapy has revolutionized the management of the acute coronary syndrome patient. In addition of reducing lowdensity lipoproteins, statins seem to induce regression of vulnerable plaques by mechanisms still under investigation. Beneficial effects have been evidenced even in normolipemic patients. Recent MRI data have evidenced statin effects on extracoronary plaque stabilization [15]

\section{Angiotensin-converting enzyme inhibitors}

Subgroup analyses of the Studies of Left Ventricular Dysfunction (SOLVD) and the Survival and Ventricular Enlargement (SAVE) chronic heart failure trials demonstrated a reduction in myocardial infarction rates in patients with left ventricular dysfunction who were treated with an angiotensinconverting enzyme inhibitor. These findings were confirmed in the landmark Heart Outcomes Prevention Evaluation (HOPE) trial that demonstrated a $22 \%$ reduction in major cardiovascular events for high-risk vascular patients treated with ramipril [16] Since these results were largely independent of the angiotensin-converting enzyme inhibitor effect on lowering blood pressure, it is postulated that there may be both direct and indirect effects on plaque stability that account for the clinical benefits realized in HOPE.

\section{Treatment of thrombosis}

Antithrombotic therapy is the cornerstone of the treatment of acute coronary syndromes (Table 2). Several pharmacologic strategies have been developed to dissolve preformed throm- 
Table 2. Regulation of thrombus formation.

\author{
LOCAL FLUID DYNAMICS \\ - Shear stress \\ - Tensile Stress \\ - Local Geometry
}

\section{NATURE OF THE EXPOSED SUBSTRATE}

- Degree of injury (mild vs. severe arterial injury)

- Composition of atherosclerotic plaque

- Residual mural thrombus

\section{SYSTEMIC THROMBOGENIC FACTORS}

- Hypercholesterolemia

- Catecholamines (smoking, cocaine, stress, etc.)

- Smoking

- Diabetes

- Homocysteine

- Lipoprotein (a)

- Infections (Chlamidia pneumoniae, Helicobacter pillory, CMV)

- Hyperchoagulable state (Fibrinogen, vWF, TF, Factor VII)

- Defective fibrinolytic state

- Emerging risk factors

CMV: Cytomegalovirus, TF: tissue factor, vWF: von Willebrand Factor

bi and inhibit thrombogenesis. They have focused on inhibiting thrombin, preventing thrombin generation, blocking initiation of coagulation, inhibiting platelet activation, or increasing fibrinolysis. These agents can be subdivided into 3 broad categories: fibrinolytics, inhibitors of the intrinsic coagulation cascade, and anti-platelet agents (Table 3). This review will focus mainly on the treatment of unstable angina and non-ST elevation myocardial infarction patients.

\section{Fibrinolytics}

Thrombolytic agents have demonstrated their effectiveness in dissolving acute thrombi, resulting in vascular reperfusion and myocardial salvage and reducing morbidity and mortality of patients with myocardial infarction. The management of acute ST-segment elevation myocardial infarction revolves around either mechanical reperfusion with primary percutaneous coronary intervention or the administration of a fibrinolytic. Fibrinolytics have undergone modifications that now allow for bolus administration with tolerable bleeding risks.

\section{Inhibitors of the intrinsic coagulation pathway} UNFRACTIONATED HEPARIN

Several well-designed clinical trials led to the widespread use of unfractioned heparin in acute coronary syndrome patients. Unfractioned heparin is an indirect thrombin inhibitor that also inhibits factor Xa. In a comprehensive meta-analysis of all acute coronary syndrome trials with unfractioned heparin, a modest risk reduction when unfractioned heparin was added to aspirin was evidenced [17]. The requirement of measuring of activated partial thromboplastin times (aPTT) and a potential bleeding risk are its major drawbacks.
Table 3. Antithrombotic therapy.

\begin{tabular}{l}
\hline ANTIPLATELET AGENTS \\
ASPIRIN \\
TICLOPIDIN, CLOPIDOGREL \\
GP IIb/IIIa RECEPTOR ANTAGONISTS \\
DIRECT THROMBIN INHIBITORS
\end{tabular}

\author{
ANTICOAGULANTS \\ - INHIBITORS OF THE INTRINSIC PATHWAY \\ HEPARIN \\ WARFARIN \\ LOW-MOLECULAR-WEIGHT HEPARINS \\ DIRECT THROMBIN INHIBITORS \\ - INHIBITORS OF TISSUE FACTOR PATHWAY \\ TFPI, TAP \\ INHIBITORS OF COAGULATION FACTORS VIIa and/or Xa
}

\section{THROMBOLYTICS}

NEW APPROACHES
P2T ANTAGONISTS (Antiplatelet)
THROMBOXANE ANTAGONISTS (Antiplatelet)
FACTOR IX INHIBITORS (Anticoagulant)
PENTASACCHARIDES (Antiplatelet + anticoagulant)

GP IIb/IIIa = glycoprotein IIb/IIIa; TFPI = tissue factor pathway inhibitor; TAP = Tick Anticoagulant Protein.

\section{Low Molecular Weight HeParin}

The development of low-molecular-weight heparin appeared to be a major advance in antithrombotic therapy for acute coronary syndromes. First introduced in the mid-1980s for the prevention of postoperative deep venous thrombosis, low-molecular-weight heparins provide a more reliable pharmacodynamic profile with a clearance and half-life that remain constant despite the dose delivered, leading to the advantage that monitoring of dosing is not generally warranted [18].

In a large meta-analysis of all trials comparing low-molecular-weight heparin versus unfractioned heparin in patients with acute coronary syndrome treated with aspirin therapy, there appeared to be a slight trend towards a lower rate of early death or myocardial infarction (odds ratio $0.88,95 \%$ confidence interval 0.69-1.12) in favor of low-molecular-weight heparin with no differences in adverse bleeding events [19]. Differences in anti-factor Xa activity between agents such as enoxaparin and daltaperin may explain discrepancies in trial results. To date low-molecular-weight heparin is being increasingly prescribed primarily for the ease and reliability of administration.

\section{The efficacy and safety of subcutaneous route}

Enoxaparin in Non-Q-wave Coronary Events (ESSENCE) and Thrombolysis in Myocardial Infarction-11B (TIMI-11B) trials confirmed that enoxaparin is superior to unfractioned heparin in reducing composite end-points (myocardial infarction and emergency revascularization) without increasing the risk of major bleeding [20]. No significant difference was 
observed in mortality, however, the meta-analysis pooling the data of ESSENCE and TIMI-11B showed a $20 \%$ reduction in death and serious cardiac ischemic events, which appeared within the first 8 days of treatment and was sustained through 43 days [20].

In the Assessment of the Safety and Efficacy of a New Thrombolytic-3 (ASSENT-3) trial [21], head-to-head comparison in patients with myocardial infarction, the combination of the fibrinolytic agent (tenecteplase) with enoxaparin demonstrated superior clinical benefit than when combined with heparin. Furthermore, the effectiveness of the combination of enoxaparin with tenecteplase was similar to that of the combination of half-dose tenecteplase and abciximab; enoxaparin, however, resulted in a significant lower incidence of major bleeding. In view of the present data and the ease of administration, enoxaparin might be considered an attractive alternative anticoagulant treatment when given in combination with tenecteplase. These results strongly suggest a better efficacy of low-molecular-weight heparins versus unfractioned heparin. Whether enoxaparin is a desirable anticoagulant in conjunction with less fibrin-specific agents or whether enoxaparin can replace unfractionated heparin in combination with a platelet glycoprotein IIb/IIIa inhibitor and what role various pharmacologic combinations will ultimately have in conjunction with early coronary intervention remains to be determined.

\section{Anti-platelet agents}

\section{ASPIRIN}

Aspirin has been the most used anti-platelet therapy for years. Several randomized multicenter trials and meta-analyses have shown the protective effect of aspirin and other antiplatelet agents. Aspirin was shown to decrease the risk of cardiovascular morbidity and mortality in primary and secondary prevention studies. Death from cardiac causes as well as fatal and nonfatal myocardial infarction were reduced by $50 \%$ to $70 \%$ in patients with acute coronary syndrome. Currently a dose of 80 to $325 \mathrm{mg}$ per day is recommended, with the understanding that higher doses of aspirin are associated with more frequent gastrointestinal side effects [22, 23]. Aspirin irreversibly blocks platelet cyclooxygenase and therefore the synthesis of the potent platelet activator thromboxane A2. However, aspirin fails to block platelet activation by other agonists such as thrombin, adenosine diphosphate or collagen; therefore, more potent anti-platelet therapies have been recently developed

\section{TRIFLUSAL}

A platelet Cox-1 inhibitor has also shown efficacy not only in coronary thrombosis but also at cerebrovascular level [24, 25]

\section{Clopidogrel}

Clopidogrel, a thienopyridine derivative, affects the adeno- sine diphosphate-dependent activation of the glycoprotein IIb/IIIa complex and effectively inhibits platelet aggregation. The clopidogrel versus Aspirin in Patients at Risk of Ischemic Events (CAPRIE) trial demonstrated that in patients with a history of myocardial infarction, stroke or peripheral artery disease, clopidogrel reduced recurrent ischemic events by almost 9\% when compared to aspirin alone [26]. The overall safety profile of clopidogrel appears at least as good as that of medium-dose aspirin. This finding led to the clinical question about the efficacy of combined aspirin and clopidogrel in a variety of cardiovascular syndromes.

The combination of clopidogrel and aspirin gives similar results to the combination of ticlopidine and aspirin in preventing coronary stent thrombosis, but is better tolerated and shows less adverse events [27]. Recently, the Clopidogrel in Unstable angina to prevent Recurrent Events (CURE) study demonstrated the superiority of the combination of clopidogrel plus aspirin versus aspirin alone in patients with acute coronary syndrome [28]. The CURE investigators reported on more than 12,000 acute coronary syndrome patients who were randomly assigned clopidogrel or placebo in addition to aspirin therapy for 3 to 12 months. The primary outcome was either death, nonfatal myocardial infarction or stroke. On long-term follow-up, the primary event rate in the clopidogrel arm was $9.3 \%$ compared with $11.4 \%$ in the placebo arm (odds ratio 0.80 , 95\% confidence interval 0.72-0.90) [28]. As well, the incidence of important secondary in-hospital events such as refractory angina, chronic heart failure and the need for revascularization was reduced in the treatment arm. Interestingly, significant benefit was achieved during the first days of treatment since the curves corresponding to the cumulative hazard rate for the combined cardiac end-point (cardiovascular death, myocardial infarction, or stroke) started to diverge in the first 3 days post-treatment and thereafter maintained throughout the study.

\section{GLYCOPROTEIN IIBIIIA INHIBITORS}

Extensive clinical trial experience from evaluating intravenous glycoprotein IIbIIIa inhibitors in patients undergoing an invasive approach to acute coronary syndrome management has led to their adoption as routine therapy. Kong and colleagues performed a meta-analysis of 16 randomized trials of glycoprotein IIbIIIa inhibitors and discovered an odds ratio of 0.76 (95\% confidence interval0.66-0.87) for the risk of death or nonfatal MI and found similar results for patients with or without ACS [29]. Various trials with different GPIIb/IIIa inhibitors demonstrated consistent clinical benefit within the context of percutaneous coronary intervention [30,31]. Unlike intravenous glycoprotein IIb/IIIa inhibitors, available data regarding any role of oral glycoprotein IIb/IIIa inhibitors are not favorable.

New antithrombotic strategies

Newly developed approaches to coronary thrombosis pre- 
vention and treatment have targeted the tissue factor pathway. Because of the key position of tissue factor as the initiator of the extrinsec coagulation pathway leading to clot formation after injury, specific anti-tissue factor antibodies, factor VIIa and $\mathrm{Xa}$ inhibitors, or the recombinant form of endogenous tissue factor pathway inhibitor [32] may afford at least a theoretical advantage over therapies that target more "downstream" components of coagulation cascade. Other approaches still in an early preclinical phase are inhibitors of glycoprotein-Ib receptor (anti vonWillebrand), inhibitors of the adenosine diphosphate receptor P2T inhibitors and NO-donors.

Several inhibitors of the tissue factor pathway have already being investigated in clinical trials. The pharmacokinetics and safety of recombinant active site site-blocked activated factor VII (FFR-rFVIIa) after single doses in healthy subjects has been recently reported [33]. In addition, the safety of increasing doses of FFR-rFVIIa is being investigated in the on-going Active Site Inhibited Seven (ASIS) trial.

Direct factor Xa inhibitors are being studies in the on-going Xa Neutralization for Atherosclerotic Disease UnderstandingAcute Coronary Syndromes (XaNADU-ACS) study. A new indirect factor $\mathrm{Xa}$ inhibitors, the heparin derivate pentasaccharide, fondaparinux (Org31540/SR90107A), has shown that it acts on the two pathways of the coagulation cascade, selective inhibition of factor Xa and high-affinity binding to antithrombin III and inhibits thrombin generation and thrombus formation and growth. A clear dose-dependent effect on the prevention of venous thrombosis, as well as on the incidence of bleeding has been reported. Several phase II and III studies have been recently completed, and fondaparinux was reported to be the first antithrombotic to demonstrate significant benefit over low-molecular-weight heparin for the prevention of venous thromboembolism in three different types of orthopedic surgery: hip fracture, hip replacement and knee surgery [34]. The overall relative risk reduction in major orthopedic surgery, in both proximal and distal deep vein thrombosis, was $50 \%$ compared with low-molecular-weight heparin.
Despite their potent anticoagulant effect and positive results in small human studies, the first-generation thrombin inhibitors have failed to provide significant beneficial outcome compared with heparin in large randomized clinical trials. In general, the administration of thrombin inhibitor shows a significant effect during the period of administration that is not maintained thereafter. Therefore, an oral form of direct antithrombin compounds allowing long-term treatment has been object of active research. The first one of this new type of oral antithrombins reaching the clinical stage is the prodrug ximelagatran (H 376/95) formed by chemical modification of melagatran. Ximelagatran showed potent direct and reversible thrombin inhibition in both animal model and human thrombosis. The orally active ximelagatran seems promising for long-term thrombin inhibition in the prevention of both venous and arterial thrombosis, and may solve the problem of the rebound phenomenon seen after discontinuation of parenteral administration of first generation thrombin inhibitors.

The clinical experience with ximelagatran so far includes more than 20,000 patients, who have been enrolled in clinical trials comparing this oral agent with low-molecular-weight heparins in the prevention or treatment of deep venous thrombosis and pulmonary embolism after major orthopedic intervention. Ximelagatran showed a relative reduction in the incidence of deep venous thrombosis and pulmonary embolism after total knee replacement [34].

\section{Acknowledgement}

The work reported in this review has been partially supported by FIS-PI020361 and PN-SAF-2000-0174. G.Vilahur is supported by a predoctoral fellowship (BEFI) from FISS and E. Segalés by a predoctoral fellowship from Fundación de Investigación Cardiovascular, Barcelona.

\section{References}

1. Badimon JJ, Fuster V, Chesebro J, Badimon L: Coronary Atherosclerosis. A Multifactorial Disease. Circulation 1993; 87: 3-16.

2. Fuster V, Badimon L, Badimon JJ, Chesebro $\mathrm{JH}$ : The pathogenesis of coronary artery disease and the acute coronary syndromes. N Engl J Med 1992; 326: 242-250 and 310-318. Parts I and II.

3. Badimon L, Badimon JJ, Fuster V: Pathophysiology of arterial thrombosis; in Gresele P, Page CP, Fuster V, Vermylen J (ed): Platelets in thrombotic and non-thrombotic disorders. Cambridge University Press, 2002, pp 727-737.

4. Corti R, Badimon L, Fuster V, Badimon JJ: Endothelium, flow, and atherothrombosis; in Fuster V (ed): Assessing and modifying the vulnerable atherosclerotic plaque. American Heart Association. Futura Publishing Compnay Inc, 2002, pp 287-305.

5. Guyton JR, Klemp KF: Development of the lipid-rich core in human atherosclerosis. Thromb Vasc Biol 1996; 16: 4-11.

6. Llorente-Cortés V, Martínez-González J, Badimon L: LDL receptor-related protein mediates uptake of aggregated LDL in human vascular smooth muscle cells. Arterioscler Thromb Vasc Biol 2000; 20: 1572-1579.

7. LLorente-Cortés V, Otero M, Hurt-Camejo E, Martínez-González J, Badimon L: Human coronary smooth muscle cells uptake versicanmodified LDL through both the low density lipoprotein receptor-related protein and the LDL receptor. Arterioscler Thromb Vasc Biol
2002; 22: 387-393.

8. Llorente-Cortés V, Otero-Viñas M, Badimon L. Differential role of heparan sulfate proteoglycans on aggregated LDL uptake in human vascular smooth muscle cells and mouse embryonic fibroblasts. Arterioscler Thromb Vasc Biol 2002; 22:1905-1911.

9. Llorente-Cortés V, Otero-Viñas M, Sánchez S, Rodríguez C, Badimon L. LDL up-regulates Low density lipoprotein receptor-related protein expression in vascular smooth muscle cells. Possible involvement of sterol regulatory element binding protein-2-dependent mechanism. Circulation 2002; 106: 3104-3110.

10. Falk E, Shah PK, Fuster V: Coronary plaque rupture. Circulation 1995; 92: 657-671.

11. Fernandez-Ortiz A, Badimon J, Falk E, Fuster 
V, Meyer B, Mailhac A, Weng D, Shah PK, Badimon L: Characterization of the relative thrombogenicity of atherosclerotic plaque components: implications for consequences of plaque rupture. J Am Coll Cardiol 1994; 23: 1562-1569.

12. Toschi V, Gallo R, Lettino M, Fallon JT, Gertz $\mathrm{SD}$, Fernández-Ortiz A, Chesebro JH, Badimon L, Nemerson Y, Fuster V, Badimon JJ: Tissue factor modulates the thrombogenicity of human atherosclerotic plaques. Circulation 1997; 95: 594-599.

13. Wilcox JN, Smith KM, Schwartz SM, Gordon $\mathrm{D}$ : Localization of tissue factor in normal vessel wall and in the atherosclerotic plaque. Proc Natl Acad Sci USA 1989; 86: 2839-2843.

14. Eriksson J, Lindstrom J, Valle T, Aunola S, Hamalainen H, Ilanne-Parikka P, KeinanenKiukaanniemi S, Laakso M, Lauhkonen M, Lehto P, Lehtonen A, Louheranta A, Mannelin M, Martikkala V, Rastas M, Sundvall J, Turpeinen A, Viljanen T, Uusitupa M, Tuomilehto J: Prevention of Type II diabetes in subjects with impaired glucose tolerance: the Diabetes Prevention Study (DPS) in Finland. Study design and 1-year interim report on the feasibility of the lifestyle intervention programme. Diabetologia 1999; 42(7): 793-801.

15. Corti R, Fayad ZA, Fuster V, Worthley SG, Helft G, Chesebro J, Mercuri M, Badimon JJ: Effects of lipid-lowering by simvastatin on human atherosclerotic lesions: a longitudinal study by high-resolution, noninvasive magnetic resonance imaging. Circulation 2001; 104(3): 249-252.

16. Yusuf S, Sleight P, Pogue J, Bosch J, Davies R, Dagenais G: Effects of an angiotensin-converting-enzyme inhibitor, ramipril, on cardiovascular events in high-risk patients. The Heart Outcomes Prevention Evaluation Study Investigators. N Engl J Med 2000; 342(3): 145-153.

17. Oler A, Whooley MA, Oler J, Grady D: Adding heparin to aspirin reduces the incidence of myocardial infarction and death in patients with unstable angina. A meta-analysis. Jama 1996; 276(10): 811-815.

18. Braunwald E, Antman EM, Beasley JW, Califf RM, Cheitlin MD, Hochman JS, Jones RH, Kereiakes D, Kupersmith J, Levin TN, Pepine
CJ, Schaeffer JW, Smith EE 3rd, Steward DE, Theroux P, Alpert JS, Eagle KA, Faxon DP, Fuster V, Gardner TJ, Gregoratos G, Russell RO, Smith SC Jr: ACC/AHA guidelines for the management of patients with unstable angina and non-ST-segment elevation myocardial infarction. A report of the American College of Cardiology/American Heart Association Task Force on Practice Guidelines (Committee on the Management of Patients With Unstable Angina). J Am Coll Cardiol 2000; 36(3): 9701062.

19. Eikelboom JW, Anand SS, Malmberg K, Weitz JI, Ginsberg JS, Yusuf S: Unfractionated heparin and low-molecular-weight heparin in acute coronary syndrome without ST elevation: a meta-analysis. Lancet 2000; 355(9219): 1936-1942.

20. Antman EM, Cohen M, Radley D, McCabe C, Rush J, Premmereur J, Braunwald E: Assessment of the treatment effect of enoxaparin for unstable angina/non-Q-wave myocardial infarction. TIMI 11B-ESSENCE meta-analysis. Circulation 1999; 100(15): 1602-1608.

21. Efficacy and safety of tenecteplase in combination with enoxaparin, abciximab, or unfractionated heparin: the ASSENT-3 randomised trial in acute myocardial infarction. Lancet 2001; 358(9282): 605-613.

22. Collaborative overview of randomised trials of antiplatelet therapy--I: Prevention of death, myocardial infarction, and stroke by prolonged antiplatelet therapy in various categories of patients. Antiplatelet Trialists' Collaboration. Bmj 1994; 308(6921): 81-106.

23. Patrono C, Coller B, Dalen JE, FitzGerald GA, Fuster V, Gent M, Hirsh J, Roth G: Plateletactive drugs: the relationships among dose, effectiveness, and side effects. Chest 2001; 119(1 Suppl): 39S-63S.

24. Cruz-Fernández JM, Lopez-Bescos L, GarciaDorado D, Lopez Garcia-Aranda V, Cabades A, Martin-Jadraque L, Velasco JA, CastroBeiras A, Torres F, Marfil F, Navarro E: Randomized comparative trial of triflusal and aspirin following acute myocardial infarction. Eur Heart J 2000; 21: 430-432.

25. McNeely W, Goa KL: Triflusal. Drugs 1998; 55: 823-833.
26. A randomised, blinded, trial of clopidogrel versus aspirin in patients at risk of ischaemic events (CAPRIE). CAPRIE Steering Committee. Lancet 1996; 348(9038): 13291339.

27. Bertrand ME, Rupprecht HJ, Urban P, Gershlick AH, Investigators FT: Double-blind study of the safety of clopidogrel with and without a loading dose in combination with aspirin compared with ticlopidine in combination with aspirin after coronary stenting: the clopidogrel aspirin stent international cooperative study (CLASSICS). Circulation 2000; 102(6): 624-629.

28. Effects of Clopidogrel in Addition to Aspirin in Patients with Acute Coronary Syndromes without ST-Segment Elevation. The Clopidogrel in Unstable Angina to Prevent Recurrent Events Trial Investigators. N Engl J Med 2001; 345(7): 494-502.

29. Kong DF, Califf RM, Miller DP, Moliterno DJ, White HD, Harrington RA, Tcheng JE, Lincoff AM, Hasselblad V, Topol EJ: Clinical outcomes of therapeutic agents that block the platelet glycoprotein IIb/IIIa integrin in ischemic heart disease. Circulation 1998; 98(25): 2829-35

30. Coller BS: Anti-GPIIb/IIIa drugs: current strategies and future directions. Thromb Haemost 2001; 86(1): 427-43.

31. Lincoff AM, Califf RM, Topol EJ: Platelet glycoprotein $\mathrm{IIb} / \mathrm{III}$ a receptor blockade in coronary artery disease. J Am Coll Cardiol 2000; 35(5): 1103-1115.

32. Badimon JJ, Lettino M, Toschi V, Fuster V, Berrozpe M, Chesebro JH, Badimon L: Local inhibition of tissue factor reduces the thrombogenicity of disrupted human atherosclerotic plaques. Effects of TFPI on plaque thrombogenicity under flow conditions. Circulation 1999; 99: 1780-1787.

33. Erhardtsen E, Nilsson P, Johannessen M, Thomsen MS: Pharmacokinetics and safety of FFR-rFVIIa after single doses in healthy subjects. J Clin Pharmacol 2001; 41(8): 880-885.

34. Eriksson BI, Bauer KA, Lassen MR, Turpie AG: Fondaparinux compared with enoxaparin for the prevention of venous thromboembolism after hip-fracture surgery. $\mathrm{N}$ Engl J Med 2001; 345: 1298-1304. 